\title{
Psychological intervention in amyotrophic lateral sclerosis: suggestions for clinical practice
}

\begin{abstract}
Objective: The present work collects considerations on the state of the art and the exchange of psychologists' professional opinions from all over Italy. Psychological support is offered along the whole progress of the disease, thus different settings are required. In this work we considered the three main areas of psychological intervention: hospital, home, help group.
\end{abstract}

Main outcome measures: Qualitative approach was used to analyze suggestions from psychologists working in the ALS field. Focus groups were used to collect data.

Results: Specific suggestions for each context were obtained collecting data from professional focus groups as the main purpose of the work.

Conclusion: The base assumption for all of the professionals is to respect each patient's need and value. Common objectives of this area are promoting awareness, improving adjustment and reducing emotional distress, together with support in communication and decision-making processes.

Keywords: neuromuscular disease, psychological practice, home assistance, support groups, hospital team care
Volume 8 Issue 2 - 2017

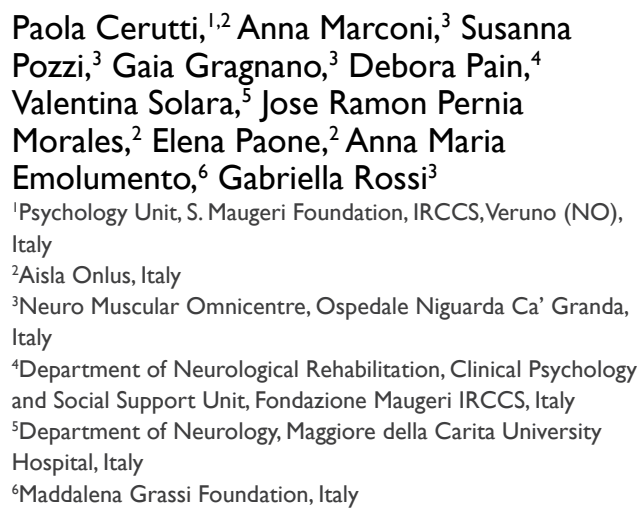

Correspondence: Anna Marconi, Neuro Muscular Omnicentre, Fondazione Serena Onlus, Piazza Ospedale Maggiore, 3, 20162 Milan, Italy, Tel 39-029|4337I, Fax 3902914337200,Email anna.marconi@centrocliniconemo.it

Received: September 05, 2017 | Published: October II, 2017

\section{Introduction}

Amyotrophic lateral sclerosis is an adult onset, fatal disorder, characterized by degeneration of both upper and lower motor neurons, muscle atrophy and weakness. Subsequently, spreading paralysis of the voluntary muscles often develops, and eventually it can also reach respiratory muscles. ${ }^{1}$ It is now well recognized that psychological counselling and support play a crucial role in ALS patients and their families. ${ }^{2-4}$ Psychosocial and emotional support for patients and caregivers is fundamental both in the moment of clinical diagnosis and during the disease progression, ${ }^{2}$ till terminal stages and death. The present study considers 24 Italian psychologists, members of the Italian Group of Psychologists for Amyotrophic Lateral Sclerosis (GIPSLA) and actively working with ALS patients in different and complementary settings: hospitals, homes, help groups. Psychologists working in hospitals help patients face the difficulty of being hospitalized and help caregivers to approach the assistance burden. Psychological assistance at home embraces the need of all the family's system. ${ }^{5}$ The Help Group represents a moment of encounter and a way to face sufferings related to the disease $e^{6}$ and is based on active participation, in that people are considered as both help suppliers and beneficiaries. The aim of the present study is to provide suggestions for psychologists working with ALS patients, defining the specific needs of the patients and caregivers in each disease stage and setting. Focus groups within GIPSLA psychologists were used for data collection. Gipsla is comprised of psychologists and psychotherapists that work for ALS centers spread throughout Italy. It is promoted and supported by AISLA, a non-profit Italian Association for ALS that guarantees the caregiving of these patients. GIPSLA's psychologists usually meet with the aim of training, discussing and sharing the best modalities to manage psychological support in such a complex field. The suggestions obtained using this qualitative approach and the expertise of this group may be considered as an example for clinicians that are dealing with ALS patients or with neurodegenerative diseases more in general.

\section{Materials and methods}

A focus group approach was used to explore the main application of psychology intervention in the ALS field. A focus group is defined as a form of group interview that explicitly uses group interaction in order to explore people's experiences, attitudes and knowledge encouraging them to talk and to engage with one another. ${ }^{7}$ Focus group discussions were described as the most appropriate method for the purposeful use of interaction to facilitate the expression of ideas, to generate meaningful opinions and suggestions ${ }^{8}$ and to explore different types of solutions. ${ }^{7}$ Convenience samplings were used and 34 GIPSLA psychologists were selected by email. 10 professional refused to participate, most of them for logistic reason especially the distance between the headquarter and their residence. The 24 participant were divided into focus groups of 8 participants, according to literature smaller groups can be effective for complex topics and especially with expert participants as in that case. ${ }^{9}$ Groups were divided according to three topics relative to the different areas of intervention: hospital care (group A), home care (group B), help group (group C). Participants were aware of the purpose of the study. Two focus group sessions were defined and structured for each of the three areas. During the first focus group session, the psychologists were asked to reflect and identify the psychologist's fundamental moment of intervention during the disease progression in each area of intervention. During the second focus group session the psychologists were asked to delineate specific suggestions, chosen during the first session, useful in clinical practice and specific for each step of the disease. This approach allowed us to compare and discuss the research and the clinical experiences in order to define suggestions addressable to professionals. The focus 
group sessions were arranged in AISLA's Milan headquarter in a non-clinical setting and a person for each area moderated the discussion, allowing continuity. The three moderators were the three main experts in each area and were recruited considering the proficiency and capability in conducting focus group sessions. Qualitative data analysis involved several readings of reports managed by three coders (trained psychologist), from which key themes and concepts were developed to obtain practical suggestions for psychologists, as reported hereafter. The main author provided a list of code for the analysis of the key themes, identified in advance from the data in order to obtain practice suggestion for psychologist in the different sessions.

\section{Results}

Data collected from the focus group sessions allowed to define the three topics of psychological support in ALS, shared among the different areas of intervention (A, B, C): initial coexistence with the disease, therapeutic decisions and dealing with end of life stages. These results represent different steps of the disease, where patients' experiences have different emotional and behavioral conditions. ${ }^{10}$ For each stage of the disease, psychological suggestions were obtained and collected considering the main topic of intervention, as follows.

\section{Group A: hospital}

The beginning: diagnostic and prognostic phases are very delicate moments, full of expectations, worries ${ }^{11}$ and beliefs, connected to perceived symptoms. ${ }^{12}$ The first psychological interview aims at assessing the following aspects: patients' emotional experiences ${ }^{13}$ relational dynamics, patients' resources, and personality traits. Emerging elements may become a useful source of information for physicians, contributing to build a more effective and customized communication process.

\section{Suggestions}

1. Generally, during diagnosis/prognosis communication, while the physiologist is concerned, individual sessions with the patient and the caregiver should be planned. In cases in which the patient is hospitalized and still hasn't received the diagnosis, the psychologist should plan individual sessions.

2. In critical situations, in agreement with the physician, the psychologist should be present during the diagnostic communication, in order to collect more information for a subsequent psychological intervention and to be able to give an emotional support.

3. Structured sessions with both the physician and the psychologist are recommendable a few days after the diagnosis communication. The psychologist assesses how the patient and his/her caregivers managed the communication and the consequent cognitive and emotional elaborations. Distorted information and expectations must be recognized in this setting and, if present, shared with the physician.

Living with the disease: Psychological sessions for inpatients are structured into a care route arranged with the medical team working on the patient's case. Sessions include different approaches: assessment interviews, psychometric and neuropsychological evaluations, psycho-educational interventions, support and mediation counselling.

\section{Suggestions}

a. It is important for the psychologist to share information of clinical cases with all of the professionals, in respect of the professional secrecy. This approach can promote therapeutic analysis of a patient case as well as a professional growth. b. The first psychological session may be followed by a psychometric assessment of the cognitive and emotional profile. Previously, both the psychologist and other co-workers should define a neuropsychological battery prototype to use, considering time availability and clinical cases.

Therapeutic decision: and end of life: dealing with end-of-life decisions is complex and distressing for both patients and relatives. ${ }^{14,15}$ Any care option in the hospital during the late stages (pharmacological, therapeutic, functional or social) should respect personal choices and individual time necessary for psychological elaboration. ${ }^{16}$ The possibility of modifying over time the decision taken is safeguarded. ${ }^{15}$

\section{Suggestions}

i. The psychologist should support patients/caregivers/families during decision-making processes, planning meetings over time, in respect of one's personal time of elaboration.

ii. The psychologist should help the physician in identifying the right time for a patient to reflect about end-of-life choices before emergency occurs.

iii. The psychologist should verify the awareness of patients and of their familiars on possible health complications. Awareness has indeed the power of influencing the patients' choices.

iv. In case of cognitive deterioration or mental disorder compromising decision making ability, the head of a legal guardianship should be recommended.

\section{Group B: home}

The beginning: psychologist's intervention at home may occur consequently to a care team's suggestion on patients'/family's request or to recommendation from trade associations. Psychologists first check the continuity of medical and social assistance, then assess needs and collect the patient's clinical history as in the hospital context, with the flexibility needed for the more informal context.

\section{Suggestions}

1. The psychologist must pay more attention on the home setting and on how to create a therapeutic alliance not only with the patient, but also with the family members.

2. The psychologist should be available for every family member, in respect of everyone's privacy.

3. An assessment session is useful also for the patient's primary caregiver.

4. Timing of meetings may be modulated over time in respect of the family's routine and clinical needs.

5. It is recommended to plan periodical meetings with the care team.

Living with the disease: the psychologist supports patients and their caregivers through the re-organization of daily living. Psychological intervention during the progression of the disease consists in one or more psychoeducational sessions, psychological support and mediation. The aim is to restrain the distress and to reinforce the resources of the context. ${ }^{17-21}$

\section{Suggestions}

a. After a primary meeting a follow up should be offered to assess psychological distress, even when a specific request for psychological support is absent. 
b. According to the family request, the psychologist can organize meetings for psychological monitoring.

c. The psychologist should promote the social support for the patients and relatives, stimulating also the maintenance of contacts beyond the relatives.

d. Periodic meetings with all of the professionals working at the domicile are recommended, in order to share information and feelings about the family dynamics and to find an individualized communication and relational strategy.

Therapeutic decisions and end of life: after hospitalization, the process of decision-making should proceed at home ${ }^{22,23}$. Doubts and fear could emerge during this time and the psychologist should evaluate the comprehension and elaboration of the information provided by the physician during the hospitalization.

\section{Suggestions}

a. Before facing this subject with the patient, the psychologist should assess family dynamics in that conflicts related to crucial decisions may emerge and involve also family members that seemed to be distant from the circumstances.

b. The psychologist must be in touch with the patient's clinicians in order to share information about end of life decisions.

c. During the stage of end of life decisions, the psychologist should incentivize the family to approach the concept of death and to speak about their fears.

d. The presence of a minor into the family requires particular attention. Specific support strategies should be provided and consent from both of the parents should be given.

\section{Group C: help group}

\section{The beginning}

It is important to highlight that Help Groups do not substitute individual psychological support or psychotherapy, which are recommended in case of severe psychopathology diagnosis or distress. ${ }^{24}$ The psychologist in this setting acts as a communication facilitator who engages patients, caregivers and families. The psychologist may also collaborate in providing information material to patients and caregivers ${ }^{25}$ and has also the possibility of involving different field experts during the meetings.

\section{Suggestions}

1. It is suggested to plan at least one clinical interview in order to assess socio-relational context and emotional state before the participation. Such a preventative measure protects the person and the group and is useful to understand needs and expectations of everyone.

2. The psychologist must evaluate the level of awareness of the disease before the beginning of the group sessions.

3. The psychologist defines group frequency (every 15/30days), duration (around 90minutes), place and amount of meetings. A defined frequency setting should be determined, possibly in a non-clinical location.

Living with the disease: the psychologist promotes empowerment and autonomy of each member with the aim of developing individual potentials using the group as the main resource.

\section{Suggestions}

a. The psychologist should always be active in an indirect way, like a director who leads a script written by the group members.

b. The psychologist promotes listening, empathy and sharing.

Therapeutic decisions and end of life: during the last stage of the disease people are unable to take part to the group sessions and the psychologist must be able to follow their last days of life.

\section{Suggestions}

1. The psychologist should support patients' and caregivers' different decisions to leave the group.

2. The psychologist should be aware of people's absence and should share information about it with the group.

\section{Conclusion}

There is a lack of research on the efficacy of psychological interventions and there is the need to develop "best practice" for psychologist working in ALS field. ${ }^{26}$ Each stage of Psychological intervention is essential; ALS requires specific moments for information and communication, together with a proper time to face the symptoms' progression. ${ }^{13}$ In this study suggestions for psychological assistance were identified considering the different disease stages and the settings of intervention following guidelines for palliative care ${ }^{26}$ Three different areas of psychological intervention were analyzed as constitutive of Italian sanitary health structures: hospital, domicile, and help group. This study highlights many shared features among these settings but also differences relative to specific moods. In each context and moment, since they have the access to personal life stories and they can offer a different comprehension of people's beliefs and behaviors and a better knowledge of patients' levels of awareness, psychologists should integrate the care team. In every case, psychological intervention has the main goal of offering presence and comfort independently from cultural or gender differences, providing empathy to all of the people involved in the disease that should be included in a multidisciplinary approach to the disease itself. ${ }^{28}$ In particular, the promptness is the peculiarity of psychological support during hospitalization that can be offered during all of the disease progression, even in critical stages. At home, particular attention is placed to family dynamics; the intervention here is characterized by the creation of a proper setting. In Help Groups the psychologist focuses on the resources of each participant; coping strategies ${ }^{29}$ are shared among the participants to face distress and to create a networking against existential isolation. ${ }^{18-30}$ During the last decade, attention to psychological approach in neuromuscular diseases increased, ${ }^{31}$ nevertheless a definite model of intervention is absent and there is a lack of guidelines in literature. This work represents the first step in finding a uniformed psychological approach for Italian ALS patients in Italy. Sharing knowledge and techniques among GIPSLA members could consent to find suggestions of interventions that could help the professionals involved in the care of ALS patients, providing a better management of patients' needs. In the future, qualitative markers could be defined to standardize the procedures of patients' care and to evaluate the service quality. Starting from this work, the group identified and shared topics that need further analysis, such as the defining of common instruments to evaluate psychological features of ALS patients and to understand the efficacy of psychological intervention. Further meetings are also necessary to create an international network that could settle proper guidelines for psychological practice in this field. 


\section{Acknowledgements}

Thanks to AISLA (Italian Association for Als patients and carers), an ongoing supervision of psychological interventions and their adherence to clinical practice and equipe work will be feasible. We are also grateful to all the psychologists of the GIPSLA for their passionate participation: Aggazio Alessandra, Belletti Monia, Bianconi Giorgia, Bracco Valentina, Bruletti Gisella, Caglioni Valentina, Calanca Chiara, Carnicelli America, Carretti Barbara,Castelnuovo Eleonora,Castiglioni Chiara, Communara Cinzia, Cortiana Giovanna, Costantino Antonella, Del Giudice Roberta, Di Gangi Giusi, Di Nicola Valeria, Febbraro Flavia, Feo Concetta, Ferullo Claudia, Fossati Federica, Fumagalli Monica, Galli Raffaella, Giudici Chiara, Laura Lazzari, Locatelli Diana, Mastro Enza, Mingardi Katri, Ninci Pier Nicola, Pallanti Michela, Pilato Alessandra, Pilcher Alessio, Pozzi Chiara, Ranaldi Valentina, Rolando Cinzia, Ronchei Massimo, Sarnelli Mariafrancesca, Sommaruga Marinella, Strozzi Michela, Tanzi Francesca, Temporin Valeria, Tringali Federica, Truffelli Romina, Valdini Francesca, Vatteroni Rita. tions.

We also thank Lucia Greco for her assistance in English transla-

\section{Conflict of interest}

No potential conflict of interest was reported by the authors.

\section{Funding}

None.

\section{References}

1. Tesauro M, Consonni M, Filippini T, et al. Incidence of amyotrophic lateral sclerosis in the province of Novara, Italy, and possible role of environmental pollution. Amyotrophic Lateral Sclerosis and Frontotemporal Degeneraton. 2007;18(3-4):1-7.

2. Bakker M, Creemers H, Schipper K, et al. Need and value of case management in multidisciplinary ALS care: A qualitative study on the perspectives of patients, spousal caregivers and professionals. Amyotroph Lateral Scler Frontotemporal Degener. 2015;16(3-4):180-186.

3. Goldstein LH, Atkins L, Landau S, et al. Predictors of psychological distress in carers of people with amyotrophic lateral sclerosis: a longitudinal study. Psychol Med. 2006; 36(6):865-875.

4. Pagnini F, Rossi G, Lunetta C, et al. Burden, depression, and anxiety in caregivers of people with amyotrophic lateral sclerosis. Psychol Health Med. 2010;15(6):685-693.

5. Creemers H, Veldink JH, Grupstra H, et al. Cluster RCT of case management on patients' quality of life and caregiver strain in ALS. Neurology. 2014;82(1):23-31.

6. Siegel DJ. The Developing Mind: Second Edition: How Relationships and the Brain Interact to Shape Who We Are. (2nd edn), The Guilford Press, USA. 2016. p.1-506.

7. Kitzinger J. Qualitative research. Introducing focus groups. British Medical Journal. 1995;311:299-302.

8. McLafferty I. Focus group interviews as a data collecting strategy. $J A d v$ Nurs. 2004;48(2):187-194.

9. Krueger RA, Casey MA. Focus groups: A practical guide for applied research. (3rd edn), Sage Publication, USA. 2000. p.1-215.

10. Kübler-Ross E. La morte e il morire. (13th edn), Cittadella Ospitalità, Italy. 2013. p.1-328.

11. Vignola A, Guzzo A, Calvo C, et al. Anxiety undermines quality of life in ALS patients and caregivers. European Journal of Neurology. 2008;15(11):1231-1236.
12. Pizzimenti A, Aragona M, Onesti E, et al. Depression, pain and quality of life in patients with amyotrophic lateral sclerosis: a cross-sectional study. Funct Neurol. 2013;28(2):115-119.

13. Simmons Z. Management strategies for patients with amyotrophic lateral sclerosis from diagnosis through death. Neurologist. 2005;11(5):257-270.

14. Boerner K, Mock SE. Impact of patient suffering on caregiver wellbeing: the case of amyotrophic lateral sclerosis patients and their caregivers. Psychol, Health Med. 2012;17(4):457-466.

15. Foley G. Loss and wellbeing in amyotrophic lateral sclerosis: challenging the consensus. Amyotrophic Lateral Sclerosis \& Frontotemporal Degeneration. 2014;15(3-4):161-162.

16. Connolly S, Galvin M, Hardiman O. End-of-life management in patients with amyotrophic lateral sclerosis. The Lancet Neurology. 2015;14(4):435-442.

17. Folgheraiter F. Community Care: Teoria e pratica del lavoro sociale di rete. Centro Studi Erickson, Italy. 1992.

18. Lulé D, Pauli S, Altintas E, et al. Emotional adjustment in amyotrophic lateral sclerosis (ALS). J Neurol. 2012;259(2):334-341.

19. Simmons Z, Bremer BA, Robbins RA, et al. Quality of life in ALS depends on factors other than strength and physical function. Neurology. 2000;55(3):388-392.

20. Pagnini F, Lunetta C, Rossi G, et al. Existential well-being and spirituality of individuals with amyotrophic lateral sclerosis is related to psychological well-being of their caregivers. Amyotroph Lateral Scler. 2011;12(2):105-108.

21. Folgheraiter F, Donati P . Community Care: Teoria e pratica del lavoro sociale di rete. (2nd edn), Centro Studi Erickson, Italy. 1991. p.1-382.

22. Folgheraiter F. Interventi di rete e comunità locali: La prospettiva relazionale nel lavoro sociale. Centro Studi Erickson, Italy. 1994. p.1-288.

23. Walsh SM, Bremer BA, Felgoise SH, et al. Religiousness is related to quality of life in patients with ALS. Neurology. 2003;60(9):1527-159.

24. Van der Hulst EJ, Bak TH, Abrahams S. Impaired affective and cognitive theory of mind and behavioural change in amyotrophic lateral sclerosis. Journal of Neurology, Neurosurgery, and Psychiatry. 2015;86(11):1208-1215.

25. Matuz T, Birbaumer N, Hautzinger M, et al. Coping with amyotrophic lateral sclerosis: an integrative view. Journal of Neurology, Neurosurgery \& Psychiatry. 2010;81(8):893-898.

26. Pagnini F, Simmons Z, Corbo M, et al. Amyotrophic lateral sclerosis: Time for research on psychological intervention?. Amyotroph Lateral Scler. 2015;13(5):416-417.

27. Bede P, Oliver D, Stodart J, et al. Palliative care in amyotrophic lateral sclerosis: a review of current international guidelines and initiatives. BMJ Supportive \& Palliative Care. 2011;1(3):343-348.

28. Miller RG, Rosenberg JA, Gelinas DF, et al. Practice parameter: the care of the patient with amyotrophic lateral sclerosis (an evidencebased review): report of the Quality Standards Subcommittee of the American Academy of Neurology: ALS Practice Parameters Task Force. Neurology. 1999;52(7):1311-1323.

29. Real RGL, Dickhaus T, Ludolph A, et al. Well-being in amyotrophic lateral sclerosis: a pilot experience sampling study. Front Psychol. 2014;5:704.

30. Tramonti F, Bongioanni P, Fanciullacci C, et al. Balancing between autonomy and support: coping strategies by patients with amyotrophic lateral sclerosis. Journal of the Neurological Sciences. 2012;320(1-2):106-109.

31. Wicks P, Abrahams S, Masi D, et al. Prevalence of depression in a 12-month consecutive sample of patients with ALS. European Journal of Neurology. 2007;14(9):993-1001. 